This item was submitted to Loughborough's Institutional Repository (https://dspace.lboro.ac.uk/) by the author and is made available under the following Creative Commons Licence conditions.

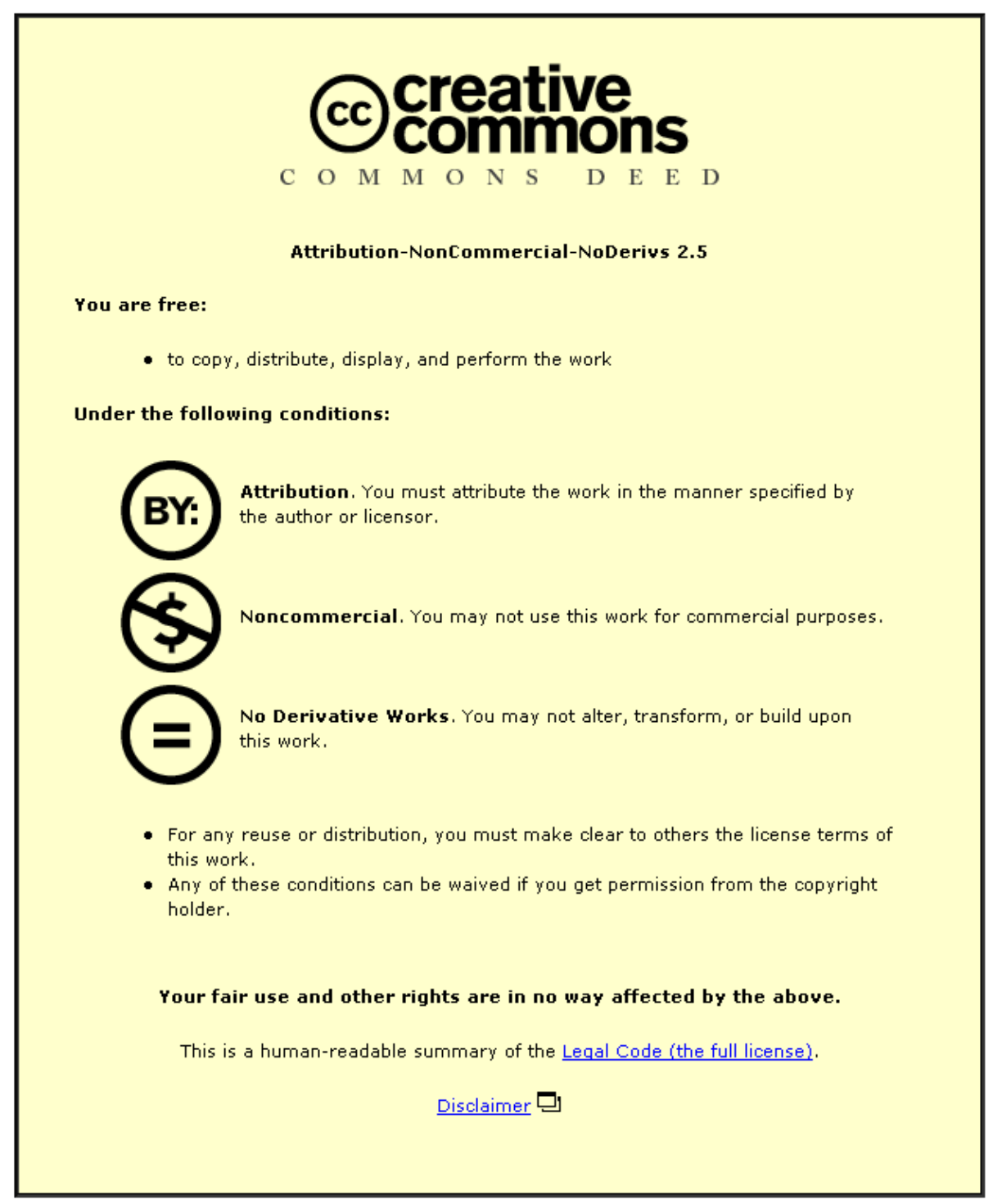

For the full text of this licence, please go to: http://creativecommons.org/licenses/by-nc-nd/2.5/ 


\section{Caffeine improves physical and cognitive performance during exhaustive exercise}

Eef Hogervorst ${ }^{1}$ MSc, PhD, Stephan Bandelow ${ }^{1}$ BA, MS, DPhil, Jeroen Schmitt ${ }^{3}$ MSc, PhD,

Roy Jentjens ${ }^{3}$ MSc, PhD, Marta Oliveira ${ }^{2}$ BSc, MSc, Judith Allgrove ${ }^{2}$ BSc, MSc,

Tom Carter ${ }^{1}$ BSc, MRes, Michael Gleeson ${ }^{2}$ BSc, PhD

Department of Human Sciences ${ }^{1}$, School of Sport and Exercise Sciences ${ }^{2}$,

Loughborough University, United Kingdom and

${ }^{3}$ Nutrition and Health Department, Nestlé Research Center, Lausanne

Corresponding author:

Prof Michael Gleeson

School of Sport and Exercise Sciences, Loughborough University,

Loughborough, Leicestershire LE11 3TU, United Kingdom

Tel. 00 44(0)15093020

Fax. 00 44(0)15093940

E-mail: E.Hogervorst@lboro.ac.uk

Running title: Caffeine and cognitive function in exercise

Financial Disclosure: This study was fully sponsored by Nestlé, Switzerland 
Caffeine and Cognitive Function during Exercise

\begin{abstract}
Caffeine is thought to act as a central stimulant and to have effects on physical, cognitive and psychomotor functioning. Purpose: To examine the effects of ingestion of a performance bar containing caffeine before and during cycling exercise on physical and cognitive performance. Methods: 24 well-trained cyclists consumed the products (performance bar containing 45 g carbohydrate and $100 \mathrm{mg}$ caffeine (CAF), isocaloric non-caffeine performance bar (CHO), or $300 \mathrm{ml}$ of placebo beverage (BEV)) at rest immediately prior to and performinged $2.5 \mathrm{~h}$ of exercise at $60 \% \mathrm{VO}_{2} \max$ followed by a time to exhaustion trial
\end{abstract} (T2EX) at $75 \% \mathrm{VO}_{2} \max$. Additional products were taken after 55 and 115 min of exercise. Cognitive function measures (computerized Stroop and Rapid Visual Information Processing tests) were performed at rest before exercise and while cycling after 70 and $140 \mathrm{~min}$ of exercise and again 5 min after completing the after the T2EX ride. Results: Participants were significantly faster after CAF when compared with CHO on both the computerized complex information processing tests, particularly after 140 min and after the T2EX ride $(\mathrm{P}<0.001)$. On the BEV trial, performance was significantly slower than after both other treatments $(\mathrm{P}<$ 0.0001). There were no speed-accuracy trade-offs $(\mathrm{P}>0.10)$. T2EX was longer after CAF consumption compared with both $\mathrm{CHO}$ and BEV trials $(\mathrm{P}<0.05)$ and T2EX was longer after CHO than after BEV $(\mathrm{P}<0.05)$. No differences were found in the ratings of perceived exertionRPE, mean heart rate, and relative exercise intensity $\left(\% \mathrm{VO}_{2} \mathrm{max} ; \mathrm{P}>0.05\right)$.

Conclusion: Caffeine in a performance bar can significantly improve endurance performance and complex cognitive ability during and after exercise. These effects may be salient for sports performance in which concentration plays a major role.

Keywords: COGNITION, CONCENTRATION, ATHLETES, CYCLING, ERGOGENIC AID 


\section{INTRODUCTION}

Paragraph 1 Caffeine is considered to be an ergogenic 'drug' with beneficial effects on both physical and mental performance and minimal side effects $(6,23)$. Oral caffeine ingestion was found to have a positive effect on time to exhaustion trials and in prolonged exercise lasting up to 2 hours $(5,7,24,28)$. While caffeine was reported to have no effect on power output after repeated bouts of very short-term intense exercise, such as 4 x 30 sec Wingate sprints with 4-min rest intervals (8, 9, $\underline{30 z 9})$, or 2 x $60 \mathrm{sec}$ maximal cycling bouts (4) or a single 30 sec Wingate test (19), others have found positive effects on exercise performance with exercise durations as brief as 4-6 $\min (3,14, \underline{30} z 9)$.

Paragraph 2 Caffeine is thought to act as a central stimulant and to have effects on cognitive and psychomotor functioning particularly during mental and physical fatigue by enhancing alertness and vigilance (16, 19, 21, $\underline{30 z 9}, 3 \underline{2} 1)$. These findings could suggest that performance enhancing effects of caffeine are due to altered central nervous system function, possibly related to attenuation of central fatigue effects $(15,25)$. Caffeine may therefore have an important role in all types of exercise in which concentration, reaction times, and technical/tactical skills have a major influence on both physical and mental performance (e.g. cycling/mountain-biking, skiing, most ball game and racquet sports, golf, orienteering, Formula 1, or in ultra endurance events).

Paragraph 3 There are many studies relating caffeine to physical performance, or relating caffeine to cognitive performance, but only few that investigated the interactions of exercise and caffeine on cognitive performance. Without caffeine, exercise itself can improve 
cognitive performance (10). For instance, one study found that a cycle ergometer endurance test at about $70 \% \dot{\vee}$ O2max improved cognitive performance in athletes, particularly on more complex cognitive tests, such as the Stroop Colour-Word test, which requires inhibition of overlearned automatic responses (12). Another study (13) revealed an additive effect of caffeine on the activating and cognition enhancing effects of exercise. $150 \mathrm{mg} / \mathrm{L}$ of caffeine in a beverage $(8 \mathrm{ml} / \mathrm{kg}$ of the beverage was consumed before exercise and $6 \mathrm{ml} / \mathrm{kg}$ was consumed during exercise) improved memory, concentration and complex reaction times in athletes after exercise and also improved exercise performance itself (an all-out $1 \mathrm{~h}$ time trial on an ergometer at $\sim 75 \%$ Wmax). Larger dosages of caffeine (225 and $320 \mathrm{mg} / \mathrm{L}$ ) had no additional effects in this study (13). Others (18) also found that caffeine (5 mg/kg) improved psychomotor performance (a multiple choice reaction time test) during exercise (multistage incremental cycling to volitional exhaustion). However, this effect, observed in 9 trained soccer players, was only seen in thermoneutral $\left(22^{\circ} \mathrm{C}\right)$ but not cold $\left(4^{\circ} \mathrm{C}\right)$ environments. Others reported no effects of a higher dose $(6 \mathrm{mg} / \mathrm{kg}$ ) of caffeine on reaction times and number recall in a mixed gender study group after two 60 sec maximal cycling bouts (4). In fact, caffeine ingestion was associated with higher blood lactate and a slower time to peak power and was suggested to have detrimental effects on anaerobic exercise performance.

Paragraph 4 Differences between the type of participants tested (gender, suboptimal training levels, caffeine habituated or caffeine naïve etc.), exercise protocols (anaerobic exercise bouts or endurance exercise / time to exhaustion trials or time trials vs. time to exhaustion), caffeine dosages and the sensitivity of the cognitive tests used (simple reaction time tests and number recall vs. more complex information processing tests) may explain differences in outcomes between studies. As far as we are aware there are no studies that have examined the effects of caffeine on both time to exhaustion and cognitive performance 
Caffeine and Cognitive Function during Exercise

during and after exercise and there are relatively few studies that have investigated whether the combination of caffeine with carbohydrate (or other macronutrients) has greater effects on physical performance and cognitive function during exercise compared with carbohydrate alone. It is well established that carbohydrate ingestion during exercise improves cognitive and endurance performance- (REF)...29).

Paragraph 5 The primary aim of the present study was to examine the effects of the ingestion of performance bars containing carbohydrate -with or without eontaining_caffeine before and during prolonged exercise on different aspects of cognitive performance during (at 70 and $140 \mathrm{~min})$ a moderate intensity cycling exercise task $\left(60 \% \dot{\vee} \mathrm{O}_{2} \max \right)$ lasting $150 \mathrm{~min}$ and after a subsequent time to exhaustion trial at a higher exercise intensity $\left(75 \% \dot{\vee} \mathrm{O}_{2} \max \right)$. To limit the number of treatment arms (taking into account the time investment interfering with training programmes) and the fact that most athletes will use some form of supplement during or just before exercise, a CHO only true placebo was used while the beverage placebo acted as a baseline measurement of cognitive and physical performance without any supplements.

\section{METHODS}

Paragraph 6 Participants. Male only subjects were recruited through personal contacts, advertisements in local sports clubs and the student pool of the University of Loughborough (UK) and/or by word of mouth. Subjects were enrolled after having fulfilled all inclusion criteria, and presenting none of the exclusion criteria_determined by both questionnaire and interview).. All participants were fully informed about the rationale for the study and of all experimental procedures to be undertaken. Participants provided written consent to 
participate in the study, which had earlier received the approval of Loughborough University ethical advisory committee.

Paragraph 7 Participants could be included if they were healthy, endurance trained male volunteers, had cycling as one of their main sports, preferably with some competitive practice and were moderate habitual caffeine users ${ }^{12}$. Participants were between $18-35$ years of age, trained at least 3 times a week for more than $2 \mathrm{~h}$ a day and had been involved in endurance training for at least 2 to 4 years. They had an aEach potential subject's aerobic capacity ( $\dot{\vee} \mathrm{O}_{2} \max$ ) was determined (as described below) during the first visit to the

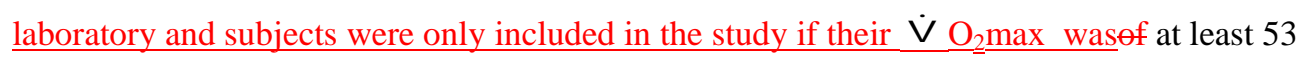
$\mathrm{ml} / \mathrm{kg} / \mathrm{min}_{\text {. }}$ and were moderate habitual caffeine users ${ }^{3}$ -

Paragraph 8 Subjects representing one or more of the following criteria were excluded from participation: Smoking or use of any (in particular psychoactive) medication that could interfere with test performance or use of illicit drugs; suffered from or had a history of cardiac, hepatic, renal, pulmonary, neurological, gastrointestinal, haematological or psychiatric illness or with any sensory or motor deficits which could be expected to affect test performance; objected to the prescription of diet (recall of diet, alcohol abstention), exercise and resting regimens or those who were not be expected to comply with treatment.

1 as derived from estimated caffeine contents in beverages and foods consumed on a weekly basis http://www.ameribev.org/industry-issues/healthy-balanced-diet/beverage-ingredients/caffeine/index.aspx

2 Due to time constraints, we had to include four participants who were caffeine naive but who were subsequently asked to increase their daily caffeine intake prior to the study (which was reflected by their increased resting salivary caffeine concentrations during the study). Two participants reported an equivalent intake of more than 6 cups of coffee a day, but all others consumed the equivalent of less than 4 cups of coffee/day.

3 Due to time constraints, we had to include four participants who were caffeine naive but who were subsequently asked to increase their daily caffeine intake prior to the study (which was reflected by their increased resting salivary caffeine concentrations during the study). Two participants reported an equivalent intake of more than 6 cups of coffee a day, but all others consumed the equivalent of less than 4 cups of coffee/day. 
Paragraph 9 Prior to the study, power-analyses were performed on the basis of our earlier work (13) using the Stroop Colour-Word test as a primary outcome measure, which indicated that $n=24$ were needed to obtain $80 \%$ power. Twenty-four healthy trained men (age $23 \pm 5$ years, body mass $73.8 \pm 8.1 \mathrm{~kg}, \dot{\vee} \mathrm{O}_{2} \max 56.6 \pm 4.7 \mathrm{ml} / \mathrm{kg} / \mathrm{min}$, power output at $\dot{\vee} \mathrm{O}_{2} \max 316 \pm 32 \mathrm{~W}$; means $\pm \mathrm{SD}$ ) were included in the present study. During the study, one participant dropped out after the first session due to time constraints and was subsequently replaced by another participant, using the same order. Upon completion, participants were paid $£ 100$ per subject and we also offered a prizes of $£ 100$ for the best overall cognitive performance and the best endurance performance (average of the 3 trials) to encourage subjects to try their best

Paragraph 10 Subjects were assigned to the treatment groups in a double-blind, placebo-controlled, randomized, cross-over, single centre, clinical trial design. All participants completed 5 exercise bouts: 2 preliminary trials $\left(\dot{\vee} \mathrm{O}_{2}\right.$ max determination and habituation trial) and 3 main trials. All trials were separated by at least 1 week.

Paragraph 11 Treatment composition. The two types of performance "energy" bars used in this study were commercially available coconut flavoured PowerBar ${ }^{\circledR}$ Performance bars and consisted of protein (5.4g), carbohydrate (44.9g), fat (3.2g), fibre (4.0g), sodium (0.6g), vitamins and minerals in amounts intended for nutritional support of athletes. The two bars were a non-caffeine $(\mathrm{CHO})$ and a caffeine containing $(\mathrm{CAF})$ version. The latter (ActiCaf $^{\circledR}$ bar) contained $100 \mathrm{mg}$ of slow release caffeine. The placebo beverage (BEV) consisted of artificial sweetened and flavoured water (Vittel Raspberry flavour) and was not intended to provide energy or nutritional ingredients.

Paragraph 12 Study design. Participants exercised under 3 different conditions: BEV trial, CAF trial or $\mathrm{CHO}$ trial. The order in which each participant undertook each condition 
Caffeine and Cognitive Function during Exercise

(BEV, CAF or $\mathrm{CHO}$ trials) was randomized and counterbalanced, where subjects were randomly assigned to one of the 6 sequences in a balanced way (CAF CHO BEV, CHO BEV CAF, BEV CAF CHO, CAF BEV CHO, BEV CHO CAF, CHO CAF BEV). Randomization was performed by TrialBalance (Nestlé program for randomization) in blocks in order to ensure balance (each treatment was applied equal times at each period). No form of stratification was applied.

Paragraph 13 Blinding. Four different codes were created for the performance bars $(\mathrm{Y}, \mathrm{Z}, \mathrm{X}$, and $\mathrm{W}$, where $\mathrm{CAF}=\mathrm{Y}$ and $\mathrm{W}$, and $\mathrm{CHO}=\mathrm{Z}$ and $\mathrm{X})$, and subjects and investigators were blinded about the content of each bar until after completion of the study. Participants were told that any of the beverages/bars could or could not contain caffeine. However, the beverage was not blinded for the investigators, who were responsible for buying and measuring quantities before administrating the blinded drinks to the volunteers. Both subjects and investigators were not allowed to discuss the experimental treatments in any format during the trials. At the end of each trial, participants completed a questionnaire to try to guess which treatment they had been given (e.g. caffeine bar, non-caffeine bar and caffeine or non-caffeine beverage) to check adequacy of blinding procedures.

Paragraph 14 Protocol prior to the treatment trials. Participants completed a food record diary for the $48 \mathrm{~h}$ period prior to habituation trial and were required to follow the same diet during the $48 \mathrm{~h}$ prior to each of the 3 main trials. Participants were required to abstain from alcohol, chocolate and heavy exercise for $24 \mathrm{~h}$ prior to each trial and to have a quiet day on the day immediately before each trial. It was also stipulated that participants should not consume any caffeine containing foods or drinks from 21:00 the night before the trial.

Paragraph 15 During the first visit an incremental cycle exercise test to volitional exhaustion was performed in order to determine the individual $\dot{\mathrm{V}} \mathrm{O}_{2} \max$ and maximal work 
rate (Wmax, the power output at $\left.\dot{\mathrm{V}} \mathrm{O}_{2} \max \right)$. These results were used to set the workload $\left(60 \% \dot{\vee} \mathrm{O}_{2} \max \right)$ for the submaximal cycle ride during the habituation and subsequent main trials. The incremental exercise test was performed on a cycle ergometer (Lode Excalibur, Groningen, The Netherlands), modified to the configuration of a racing bicycle with adjustable saddle height and handlebar position. Subjects began with a 3-min warm-up at 95 $\mathrm{W}$, followed by an incremental exercise protocol of $35 \mathrm{~W}$ every 3 min until exhaustion. These results were used to set the workloads in the experimental trials. Expired gas samples (1-min collection period) were collected into Douglas bags during the final minute of each stage for the determination of oxygen consumption and carbon dioxide production. A Polar heart rate monitor (Vantage NV, Polar, Kempele, Finland) was used for continuous recording of the heart rate (HR). Standard measures of height, body mass were also performed during this visit.

Paragraph 16 A week before the actual trial, a habituation trial was carried out which was similar to the main trials, except that no treatments were given during this trial, only water. The purpose of this habituation trial was threefold: firstly, to familiarise the participants with the physical stress of the exercise and with the methods involved in the trial; secondly, to ensure that the participants were able to maintain the selected intensity for 150 min after an overnight fast; and thirdly, to ensure that the selected work rate actually did elicit a relative intensity of $60 \% \dot{\vee} \mathrm{O}_{2} \max$.

Paragraph 17 Protocol during treatment trials. Volunteers arrived at the laboratory at 08:30 on the morning of the main trials after an overnight fast ( $>10 \mathrm{~h}$ ). After a 5 min seated rest, a blood sample was obtained from an ear lobe, using an autoclick lancet, and the blood glucose concentration was analysed using an Accutrend GC (Roche, Germany) blood glucose 
analyser. After that, unstimulated saliva samples were obtained by having subjects dribble saliva into a collection tube for $3 \mathrm{~min}$.

Paragraph 18 Participants were asked to go to the toilet and empty their bladder, and after that they had their body mass measured. Following that, participants, performed a series of cognitive tests (immediate recall of the memory test and the Visual Search test, Stroop Colour-Word test and Rapid Visual Information Processing (RVIP) test) and completed a caffeine side-effect questionnairesubjective rating scales, while seated on the cycle ergometer, with the computer screen not more than one metre away from them. To minimize external potential noise, a pair of ear plugs was used during all cognitive tests batteries, in all trials. Participants then consumed their first experimental product (performance bar or beverage) within 5 min, and performed 150 min of exercise at $60 \% \dot{\vee} \mathrm{O}_{2}$ max immediately after the consumption of the product. Further experimental supplementation of either CAF, $\mathrm{CHO}$, or $300 \mathrm{ml}$ of $\mathrm{BEV}$ was given at 55 and $115 \mathrm{~min}$. In addition, $200 \mathrm{ml}$ of water was consumed at the onset and every 20 min during the exercise bout for all trials.

Paragraph 19 During exercise, the following measurements (as illustrated in Figure 1) were taken: A caffeine side-effect questionnaire and the cognitive performance tasks (Stroop Colour-Word test and RVIP test) were administered at 70 and $140 \mathrm{~min}$. The caffeine side-effect questionnaire was developed on the basis of common and known side-effects after administration, withdrawal and overdosing of caffeine which were categorized as nervous system, gastrointestinal, pulmonary and cardiovascular effects. The questionnaire was computerized and presented before each cognitive test trial. The total number of side-effects per trial were reported. Rating of perceived exertion (RPE) was assessed during exercise every 20 min, using a paper version of the 0-20 Borg Scale (2). Ear lobe blood drawing (for glucose analysis as described above) and saliva sampling (before cognitive test sessions) 
were carried out at 65 and 125 min. Oxygen uptake, carbon dioxide production and respiratory exchange ratio (during exercise at 30, 90 and $115 \mathrm{~min}$ ) were assessed using a Douglas Bag collection system, Servomex gas analyzer and Harvard dry gas meter and HR (every 10 min) was monitored using a Polar HR monitor (Vantage NV, Polar, Kempele, Finland).

Paragraph 20 At the end of the 150 min of exercise subjects were given a 5-min break, immediately followed by a time to exhaustion trial at $75 \% \dot{\vee} \mathrm{O}_{2}$ max. Participants were instructed to maintain a pedal cadence of more than $50 \mathrm{rev} / \mathrm{min}$ while cycling, to remain seated at all the time and to attempt to cycle for as long as possible. However, no external encouragement was given during the trial. No music was played and no clues about elapsed time, power output and HR were given during the ride to exhaustion. Heart rate was recorded unobtrusively every 5 min during the ride to exhaustion. Immediately after completion of the exercise to exhaustion trial, a saliva sample and an ear lobe blood sample (for glucose analysis) were collected. Then, the caffeine side-effect questionnaire was completed and cognitive performance tasks (delayed recall of the memory test, Visual Search test, Stroop Colour-Word test and RVIP test) and subjective rating scales and questionnaire assessments were carried out. Finally, participants were weighed again to determine their weight loss. Sweat loss was estimated based on weight loss corrected for fluid intake and any urinary losses. All saliva samples collected were stored frozen at $-20^{\circ} \mathrm{C}$ prior to analysis of caffeine concentration (Emit caffeine assay kit, Dade Behring Ltd., Milton Keynes, UK).

Paragraph 21 Definition of cognitive measures. Subjects were asked to complete a cognitive test battery which lasted for approximately 15-20 min. The cognitive tests were selected based on their proven sensitivity to effects of exercise in combination with caffeine (13). 
Paragraph 22 Stroop Colour Word Test. This test was the primary outcome measure. The test measures the sensitivity to interference and the ability to suppress an automated response (time needed to read the colour words rather than the time it takes to name the colour of the letters) (27). The baseline level contained 15 stimuli (reading colour names printed in white on a black background), the colour-interference level (naming the font colour rather than reading the printed colour name, which was always incongruent) comprised 40 stimuli. Each colour word was placed on the centre of the screen with the target and distractor presented at random on the left or right side of the stimulus word, with target position counterbalanced for left and right side within each test level. The participant was required to press the left or right arrow key as quickly as possible to indicate the position of the target word. Correct response times were recorded in msec. Errors were also recorded for error analysis and to assess speed-accuracy trade-offs. Both tests started with 5 learning trials that included feed-back.

Paragraph 23 Rapid Visual Information Processing (RVIP) task. The RVIP (11) is a continuous performance test lasting 5 min where subjects are required to monitor a continuous stream of digits (100 digits per min or $600 \mathrm{msec}$ per digit) for target sequences, which were defined as 3 consecutive odd or even numbers (e.g., 3-5-7 or 2-8-4). The test had 8 targets per min with 5-30 digits between each target, and the screen was updated with a new digit every 600 msec. Subjects responded to target strings by pressing a button box linked to the parallel port. Correct detections ("hits") of target strings could be registered during the last digit of a sequence and in the subsequent 1500 msec. Reaction times (RTs) in msec, correct detections and omission errors (missed responses to targets) and commission errors (false alarms to non-target) were recorded. Analysis was performed on RTs for correct responses, true positive rate $(\mathrm{TP} /(\mathrm{TP}+\mathrm{FP}))$ and miss rate $(\mathrm{FN} /(\mathrm{FN}+\mathrm{TP}))$. 
Paragraph 24 Visual Search Test. The computerized visual search and detection test required the disengagement from one focal area of attention to locate the next target (a triangle formed from constantly moving dots). Participants were instructed to press a key as soon as they could detect a triangle on the screen. The baseline level contained 15 stimuli, which were drawn in solid green lines on a black background. In the 40 complex level stimuli, moving random dots covering the entire screen served as background distractors. New target triangles were initially drawn with just a few visible dots of each line, and the density of these points increased linearly with time until a key press response was registered. The screen was re-drawn every 250 msec. After each response, new targets appeared with random delays of at least $500 \mathrm{msec}$. Visual search results were recorded with reaction times (in msec) and the number true and false positives (1). This test was only performed before and after exercise (after the time to exhaustion trial).

Paragraph 25 Word Learning Test. This test consisted of 4 parallel (including 1 training version) word learning lists which were based on modified versions of the Hopkins, Rey and Californian Auditory Verbal Learning Tests. Each test consisted of a list of 16 monosyllabic words, which were presented in three trials on a computer screen. Items were presented in the same sequence at a rate of one per 2 sec (presentation time 1 sec, interstimulus interval $1 \mathrm{sec}$ ). Each trial ended with a free immediate recall of the words which was done before exercise. The main outcome variables of this test were the number of words correctly recalled after exercise, constituting a measure of consolidation efficiency.

Forgetting rate was calculated as the maximum number of words immediately recalled in one trial - the delayed recall .

Paragraph 26 Data Analysis. Overall treatment effects (including treatments BEV, CAF and $\mathrm{CHO}$ ) were analysed via repeated-measures ANCOVAs that included main effect 
of treatment, time, visit day, and treatment by time interactions. All analyses were performed with R (version 2.5) (www.r-project.org). If a significant treatment by time interaction was found, the responses at 70, 140 and 180 min were analysed using mixed effects models. These models contained fixed main effects of treatment, fixed main effect for mean scores at time 0 (to adjust for baseline speed) and a fixed main effect for visit day (to account for learning between visits). The nlme package was used to build linear mixed effect (lme) models for the normally distributed RT data. The lme4 package was used to build the same models for binomial data such as the proportion of correct answers, which were modelled via the binomial distribution. The resulting adjusted p values for the contrasts CAF-CHO, BEVCAF and BEV-CHO are listed on Figures $2-4$ as CCO, CAF and CHO, respectively. Significance codes of the post-hoc comparisons (adjusted for 3 comparisons at each time point) are * for $\mathrm{P}<0.05$, ** for $\mathrm{P}<0.01$ and $* * *$ for $\mathrm{P}<0.001$. For these post-hoc tests, the correlations between the $\mathrm{BEV}, \mathrm{CHO}$ and $\mathrm{CAF}$ samples were found to be around 0.5 , hence the adjusted Bonferroni-corrected alpha level for 3 comparisons used was 0.03 .

Paragraph 27 To compare the physiological and other responses (HR, maximum HR, RPE, blood glucose concentration, time to exhaustion, sweat loss, and $\% \dot{V} \mathrm{O}_{2}$ max) between the 3 different treatments (CAF, CHO and BEV) repeated measures general linear models were employed. Post hoc analyses were carried out, where appropriate, using Bonferroni corrections. All statistical analyses were carried out blinded to treatment by the on-site investigators (caffeine in saliva was analyzed last).

\section{RESULTS}

Paragraph 28 Caffeine consumption. On the basis of self-report, habitual caffeine consumption (including coffee, tea, chocolate, cola, etc. using standard estimate tables $\underline{\text { (see }}$ footnote 1REF)) was estimated to be on average $170 \mathrm{mg} /$ day (Median=121, SD $167 \mathrm{mg} /$ day), 
indicating that most participants were low to moderate caffeine consumers, ingesting the equivalent of 1-3 cups of coffee a day.

Paragraph 29 Stroop Colour-Word Test. The main outcome measure was theeomputerized Stroop Colour Word test measuring interference susceptibility. Cognitiveeffects of treatment were tested at baseline, after 70 and 140 min during exercise and at approximately $180 \mathrm{~min}$, after the time to exhaustion trial.The RTs were log-transformed to approximate a normal distribution more closely and t. The means and 95\% confidence intervals of these log-transformed response latency and accuracy, split for time, treatment, and test type are displayed in Figures 2a and 2b.

Paragraph 30 In the ANOVA of the log-transformed RT data, there was a main effect of treatment $[F(2,46)=45.7, P<0.0001]$, a main effect of time $[F(2,46)=60.9, P<0.0001]$ and an interaction between time and treatment $[F(2,46)=46.8, \mathrm{P}<0.0001]$. However, the 3way interaction between treatment, time and test type (Reading speed vs. Colour-Naming speed) was not significant $[\mathrm{P}=0.13]$. Thus, the treatment effect occurred similarly on the baseline and the colour-interference level of the Stroop test (compare Figures $2 \mathrm{a}$ and $\mathrm{b}$ ), implying that treatment effects were not specific to the interference component.

Nevertheless, we found a trend towards -significance for thę 2-way interaction between test type and the CCO treatment contrast at $180 \min [t(23)=2.13, P=0.03]$, as well as a trend $\underline{\text { towards }}$-significance for thet interaction between test type and the CAF treatment contrast $[\mathrm{t}(23)=1.84, \mathrm{P}=0.07]$. Although these significance levels do not survive the correction for 2 comparisons, the trends indicate that the caffeine effect was more pronounced on the complex interference RTs than the baseline test RTs.

Paragraph 31 Post-hoc testing revealed that participants were highly-significantly faster after treatment CAF when compared with both other treatments at times 70, 140 and 
180 min, on both levels of the test (Figure 2). RTs after treatment CHO were significantly faster than after placebo (BEV) only on the interference level, and only at 140 min (Figure 2). Performance with CAF maintained its fast response times after the time to exhaustion trial, indicating improved response latencies to complex stimuli with caffeine during and after endurance exercise. When the mean differences between the treatments were re-transformed into milliseconds via the exponential function, we found that after correcting for baseline speed, the effect size on the colour-interference test level of the CAF-CHO (CCO in Figure 2) contrast was 79 msec and the effect size of the BEV-CAF (CAF in Figure 2) contrast was 80 msec, with responses after CAF always being faster. On the baseline level of the Stroop test, the effect sizes of the CAF-CHO and CAF-BEV contrasts were 34 and $37 \mathrm{msec}$, respectively.

Paragraph 32 The overall ANOVA model of Stroop accuracy (proportion of correct responses) revealed no significant main effect of treatment $[\mathrm{P}=0.12]$, no significant treatment by time interaction, and no significant 3-way interaction between treatment, time and test type [both $\mathrm{P}>0.5$ ]. Since treatment effects were absent, no post-hoc testing was performed on the accuracy data. There was thus no speed-accuracy trade-off where athletes would have sacrificed accuracy for faster speed of performance.

Paragraph 33 Rapid Visual Information Processing task (RVIP). Gognitive effects of treatment on the RVIP were tested at baseline, after 70 and 140 min during exercise and at approximately $180 \mathrm{~min}$, after the time to exhaustion trial. The RTs were log-transformed toapproximate a normal distribution more closely. The RTs were log-transformed to approximate a normal distribution more closely and the means and 95\% confidence intervals of these log-transformed response latencies and accuracy (misses and true positive (TP) rate), split for time and treatment are displayed in Figures 3a-c. 
Caffeine and Cognitive Function during Exercise

Paragraph 34 In the ANOVA of the log-transformed RT data, there was a main effect of treatment $[\mathrm{F}(2,46)=23.3, \mathrm{P}<0.0001]$, a main effect of time $[\mathrm{F}(2,46)=59.2, \mathrm{P}<0.0001]$ and an interaction between time $\mathrm{x}$ treatment $[\mathrm{F}(2,46)=16.8, \mathrm{P}<0.0001]$. The TP rate also showed a significant main effect of treatment $[F(2,46)=10.0, P<0.0001]$, no significant main effect of time $[\mathrm{P}=0.12]$ and a significant interaction between time and treatment $[F(2,46)=3.4, P=0.03]$. Finally, the miss rate showed a highly significant main effect of treatment $[\mathrm{F}(2,46)=30.2, \mathrm{P}<0.0001]$, a highly significant main effect of time $[\mathrm{F}(2,46)=$ 26.1, $\mathrm{P}<0.0001]$ and a highly significant interaction between time and treatment $[\mathrm{F}(2,46)=$ 26.6, $\mathrm{P}<0.0001]$.

Paragraph 35 Since all outcomes showed significant treatment by time interactions, post-hoc testing was performed on all measures, the results of which are displayed in Figures 3a-c. These findings are in line with those of the Stroop mentioned above in that caffeine improved complex and rapid visual information processing during and after exercise.

Paragraph 36 On all parameters of this test, performance was better with CAF than both $\mathrm{CHO}$ and BEV. This effect emerged after 70 min of exercise for TP rate and miss rate, and after 180 min for the RT latencies (CAF-CHO, see Figure 3a). This indicates improved performance on rapid visual information processing both during and after time to exhaustion exercise with caffeine.

Paragraph 37 Visual Search Test. The RTs for correct responses were logtransformed to approximate a normal distribution more closely. The ANOVA of the logtransformed RT data revealed a significant interaction between time and treatment $[F(2,46)=$ $5.4, \mathrm{P}=0.004]$. No significant treatment effects were found on the visually simple level of this test, implying that treatment effects were specific to the visual complexity component. The means and 95\% confidence intervals of these log-transformed response latencies and 
proportion of correct responses on the complex level of the visual search test, split for time and treatment, are displayed in Figures 4a and 4b.

Paragraph 38 Post-hoc testing revealed significant treatment effects on the complex level of the visual search test. Significant response speed improvements were found at 180 min for CAF compared with BEV and $\mathrm{CHO}$ (but not for $\mathrm{CHO}$ compared with BEV) (Figure 4a). These findings indicate that the caffeine-related improvements on the visual search test are related to improved processing of complex visual scenes, and not just simple motorrelated response speed.

Paragraph 39 The overall ANOVA model of visual search test accuracy (proportion of true positives) revealed a significant treatment by time interaction $[F(2,46)=6.8, P=$ 0.001], and a significant 3-way interaction between treatment, time and test type $[F(2,46)=$ 3.1, $\mathrm{P}=0.04]$. This indicates that TP rates were affected by treatment on the complex level of this test only. Post-hoc analyses revealed no significant treatment effects on the simple test level, and significant accuracy improvements on both bars (CHO and CAF) compared with BEV (Figure 4b). However, there was no significant difference between $\mathrm{CHO}$ and $\mathrm{CAF}$ at 180 min.

Paragraph 40 It thus appears that for all outcome measures on the complex level of Visual Search Test, CAF improved performance significantly compared with BEV, and that for accuracy there was also a significant improvement with CHO compared with BEV. Furthermore, CAF produced significant response speed improvements compared with CHO. These results indicate significant performance improvements with caffeine in complex and thallenging visual environments.

Paragraph 41 Word Learning Test. The delayed recall component of this test was only performed after exercise. For these analyses repeated measures general linear models 
were used with Bonferroni corrected pairwise comparisons. Overall, within subjects effects showed no main effect of treatment $[F(2,46)=1.52, P=0.22]$. Delayed recall after exercise was non significantly higher after CAF (10.0 words recalled on average, SD=3.55) compared with treatment BEV (8.9 words recalled, $\mathrm{SD}=4.07$, non corrected paired t-test $\mathrm{P}=0.08$, Bonferroni adjusted comparisons $\mathrm{P}=0.23$ ) with performance after consumption of $\mathrm{CHO}$ having an intermediate position (9.8 words recalled, $\mathrm{SD}=3.65)$ between placebo $\mathrm{BEV}$ and CAF. Power for these secondary analyses, however, was insufficient (31\%). There was no difference in immediate recall before exercise $(\mathrm{P}>0.55)$.

Paragraph 42 Caffeine side-effects. There were no serious side-effects reported after any of the treatments. Most complaints were probably related to the effects of exercise itself, as there was no significant difference $[\mathrm{F}(2,46)=1.49, \mathrm{P}=0.24$, power $26 \%]$ between the total number of side-effects reported between treatments. For CAF, an average number of 2.1 $(\mathrm{SD}=3.3)$ side-effects was reported, for $\mathrm{CHO} 1.2(\mathrm{SD}=2.2)$ and for $\mathrm{BEV} 1.9(\mathrm{SD}=3.1)$ sideeffects. Over all trials, the most common complaints were having: a stuffy nose (20x), a dry mouth (15x), tiredness (15x), muscle trembling (12x), weakness (11x), hunger (8x), shakiness/jitters (8x) and drowsiness (7x). Less common was: the urge to urinate (4x), loss of appetite, dehydration, and irritability (all 3x), belching, dizziness and headache (all 2x), confusion, increased pain sensitivity, restlessness, cold sweats, feeling bloated, nauseous and stomach upset (1x).

Paragraph 43 Un-blinding. Blindness checks were made after exercise. After consumption of CAF, 83\% ( $\mathrm{n}-20$ ) was under the impression of having received caffeine inthe bar. Comments made by participants after the trial suggested it was the mental alerting effect ('It feels like I had my morning cup of tea'; 'I feel more awake'; 'I am more encentrated'; 'I felt like I could cycle forever') that rendered these result, but not the taste. 
Caffeine and Cognitive Function during Exercise

Only $12.5 \%(n-3)$ thought the beverage contained caffeine. Comments made were 'I have no energy'; 'I feel weak'; 'It takes a long time'; 'This surely has no caffeine in it' etc. suggesting that this placebo was insufficiently blinded. 50\% $(n=12)$ of those having consumed CHO thought it had contained caffeine, suggesting that this was well blinded.

\section{Physiological Parameters.}

Paragraph $4 \underline{3} 4$ Heart Rate. There were no significant differences in the mean HR between trials $[\mathrm{F}(2,46)=2.05, \mathrm{P}=0.14$, power $40 \%$, Bonferroni corrected comparison between $\mathrm{CAF}$ and $\mathrm{BEV}, \mathrm{P}=0.17$ ] during the 150 min rides at $60 \% \dot{\vee} \mathrm{O}_{2}$ max . However, a significant difference was found in the maximum HR achieved in the time to exhaustion trial, comparing CAF and BEV treatments $[F(2,46)=8.13, P=0.001,95 \%$ power $]$, see Table 1 .

Paragraph $4 \underline{4} 5$ Rating of perceived exertion (RPE). No significant difference in the rating of perceived exertion (RPE) was found between treatments $(\mathrm{P}>0.05)$. Thus, caffeine did not affect mean RPE during the 150 min rides at $60 \% \dot{V} \mathrm{O}_{2}$ max. The means of the RPE were $12 \pm 1,12 \pm 1$ and $13 \pm 1$ for CAF, CHO and BEV trials respectively (means $\pm \mathrm{SD}$ ). Power for these analyses was insufficient at $36 \%$. As expected, a significant effect of time was found with RPE increasing across time in all three trials $(\mathrm{P}<0.05)$, but no differences in the RPE between trials were found at any time point $(\mathrm{P}>0.05)$.

Paragraph $4 \underline{6} 6$ Sweat loss. An average sweat loss (weight loss after correction for fluid intake and any urinary losses) of $2.6 \mathrm{~kg}( \pm 0.5), 2.4 \mathrm{~kg}( \pm 0.5)$ and $2.5 \mathrm{~kg}( \pm 0.5)$ was found in the CAF, CHO and BEV trial, respectively. However, no significant differences in sweat losses were found between trials.

Paragraph $4 \underline{6} 7$ Time to exhaustion. Performance time in sec was assessed using a time to exhaustion protocol at $75 \% \dot{\vee} \mathrm{O}_{2}$ max which was introduced after a 5-min break after 
having cycled for 150 min at $60 \% \dot{\vee} \mathrm{O}_{2}$ max. There was a significant effect of treatment on time to exhaustion $[\mathrm{F}(2,46)=12.06, \mathrm{P}<0.001$, power $99 \%]$, with CAF showing the best results. Time to exhaustion in both the $\mathrm{CAF}$ and $\mathrm{CHO}$ trials was significantly longer than the $\mathrm{BEV}$ trial $(\mathrm{P}=0.001$ and 0.031 , respectively). Overall, caffeine improved the time to exhaustion by 354 sec, a 27\% improvement in performance compared with ingesting the bars without caffeine $(\mathrm{t}(23)=2.36, \mathrm{P}=0.03$, which remained significant after Bonferroni-Holm correction) and a 751 sec (84\%) improvement compared with taking a liquid beverage containing no energy source (Figure 5). Time to exhaustion was 397 sec (44\%) longer on the CHO trial than on the BEV trial $(\mathrm{P}<0.01)$.

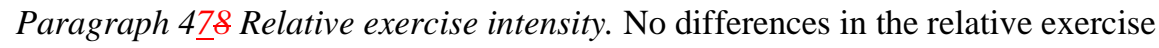
intensity $\left(\% \dot{\vee} \mathrm{O}_{2} \max \right)$ were found between trials $(\mathrm{P}=0.15$, power $=39 \%)$, and therefore all trials were done at an intensity of around $60 \% \dot{\vee} \mathrm{O}_{2} \max \left(\mathrm{CAF}=60.0 \pm 2.4 \% \dot{\vee} \mathrm{O}_{2} \max\right.$; $\mathrm{CHO}=59.0 \pm 2.5 \% \dot{\mathrm{V}} \mathrm{O}_{2} \max ;$ and $\left.\mathrm{BEV}=59.0 \pm 2.3 \% \dot{\mathrm{V}} \mathrm{O}_{2} \max \right)$.

\section{Paragraph $4 \underline{8} 9$ Respiratory Exchange Ratio and Estimated Rate of Carbohydrate}

Oxidation. Although rates of oxygen uptake were not different between the two performance bar trials (with and without caffeine), the RER and the calculated rate of carbohydrate oxidation (Table 2) were higher at 30 min of exercise in the CAF trial compared with both CHO and BEV trials $(\mathrm{P}<0.05)$. At 90 and 115 min of exercise the RER and rate of carbohydrate oxidation were both lower in trial BEV compared with the CAF and $\mathrm{CHO}$ trials $(\mathrm{P}<0.05)$.

Paragraph $\underline{49} 50$ Blood glucose concentration. Significant main effects of trial $(\mathrm{P}=$ $0.01)$, time $(\mathrm{P}<0.001)$ and a trial $\times$ time interaction $(\mathrm{P}=0.015)$ were observed for blood glucose concentration (Table 3). Both CAF and CHO trials were significantly different from trial BEV. There was no difference in the glucose concentration at pre-exercise between any 
of the trials. However, glucose concentration was significantly higher at $65 \mathrm{~min}$ in trial $\mathrm{CHO}$ compared with trial BEV $(\mathrm{P}=0.015)$, and at $125 \mathrm{~min}$ in both trials CAF and CHO compared with trial BEV $(\mathrm{P}<0.05)$. Additionally, glucose concentration increased at the end of the time to exhaustion test in the $\mathrm{CAF}$ and in the $\mathrm{CHO}$ trials compared with the $\mathrm{BEV}$ trial $(\mathrm{P}=$ 0.001). Analysing the trial $x$ time interaction, the glucose concentration in the CAF trial showed a significant difference at time to exhaustion compared with rest and with 65 min (P $=0.046$ and 0.009 , respectively). No difference was found in glucose levels between any time points for trial $\mathrm{CHO}$. In trial BEV, glucose concentration was significantly lower at 65 and 125 min of exercise $(\mathrm{P}=0.015)$ compared with pre-exercise, but this was not significantly different from rest at the end of the ride to exhaustion $(\mathrm{P}>0.05$, Table 3).

Paragraph $5 \underline{01}$ Saliva caffeine concentration. Caffeine concentrations in saliva were substantially increased in the CAF trial (Rest $=0.25 \pm 0.32 \mu \mathrm{g} / \mathrm{ml}$ to $5.93 \pm 2.84 \mu \mathrm{g} / \mathrm{ml}$ at the end of the ride to exhaustion) compared with rest values, while no changes from rest values were found in either the CHO or BEV trials (Rest $=0.14 \pm 0.22 \mu \mathrm{g} / \mathrm{ml}$ to $0.03 \pm 0.03 \mu \mathrm{g} / \mathrm{ml}$ and $0.26 \pm 0.41 \mu \mathrm{g} / \mathrm{ml}$ to $0.06 \pm 0.06 \mu \mathrm{g} / \mathrm{ml}$, CHO and BEV trials, respectively) at any time point.

Paragraph 51 Un-blinding. Blindness checks were made after exercise. After consumption of CAF, 83\% ( $\mathrm{n}=20)$ of the participants were under the impression of having received caffeine in the bar. Comments made by participants after the trial suggested it was the mental alerting effect ('It feels like I had my morning cup of tea'; 'I feel more awake'; 'I am more concentrated'; ' I felt like I could cycle forever') that rendered these result, but not the taste. Only $12.5 \%(n=3)$ thought the beverage contained caffeine. Comments made were 'I have no energy'; 'I feel weak'; 'It takes a long time'; 'This surely has no caffeine in it' etc. 
Caffeine and Cognitive Function during Exercise

suggesting that this placebo was insufficiently blinded. 50\% ( $\mathrm{n}=12)$ of those having

consumed $\mathrm{CHO}$ thought it had contained caffeine, suggesting that this was well blinded.

\section{DISCUSSION}

Paragraph 52 The present study showed better concentration, faster response speed and detection and improved complex rapid visual information processing with caffeine given in a performance bar both during moderate intensity cycling exercise (150 min at $60 \% \dot{\vee} \mathrm{O}_{2}$ max) and after a time to exhaustion trial at $75 \% \dot{\vee} \mathrm{O}_{2}$ max in trained athletes compared with a performance bar without caffeine. These alerting, focusing and vigilance improving effects are vital in most competitive sports. Memory was not improved, but our earlier study (13) had included a more complex memory test which had an interference component added to this. Pure effects of caffeine on memory performance are controversial (26). In all likelihood effects of caffeine will only occur when complex memory tests are used, which require inhibition of interference and are thus mediated by caffeine's effects on concentrational ability_(26). In addition, performance of the time to exhaustion trial itself was improved and, accordingly, maximum HR achieved during the time to exhaustion trial was higher after consumption of the caffeine containing bar. However, perceived effort was not altered and there were no significant differences between the CAF, CHO and BEV trials in relation to most of the physiological measurements, such as HR during exercise, RPE, oxygen uptake, and sweat loss. Importantly, there were no significant or major side-effects after consumption of the bar containing caffeine

Paragraph 53 An earlier study also showed improved physical and mental performance after $150 \mathrm{mg} / \mathrm{L}$ of caffeine in a carbohydrate solution (13). The present study 
indicates that caffeine given in a performance bar shows comparable effects. Similar to the earlier study (13) effects were most clearly observed on the more complex cognitive tests, and less so on tests using simple response times, indicating centrally mediated and not merely peripheral sensorimotor improvements. Some cognitive tests are thus more sensitive to the effects of caffeine with carbohydrates than others. In the same vein, two other studies (16, 321) also found improved performance on the RVIP test using low dose caffeine (33 mg and $1 \mathrm{mg} / \mathrm{kg}$, respectively) in combination with carbohydrates. It is unclear whether similar effects occur when caffeine is taken without carbohydrates. However, one of these studies (321) suggested that reversal of withdrawal effects was the major effect of caffeine on cognition and mood, as that study could not show caffeine effects when moderate caffeine consumers were no longer caffeine deprived (i.e. after they had been given a second dose of caffeine). In the present study, $100 \mathrm{mg}$ of caffeine was given 3 times at regular time intervals during the trials but cognitive performance continued to improve over time. These findings would indicate that results are not primarily explained by caffeine withdrawal alleviating effects.

Paragraph 54 Lower blood glucose concentration was found during and following exercise in the BEV trial compared with other two trials. This suggests that the consumption of an energy bar containing carbohydrate helped maintain blood glucose concentration during exercise, which otherwise falls in the absence of carbohydrate intake. Although blood glucose concentrations were not different between the two performance bar trials (with and without caffeine), the rate of carbohydrate oxidation was higher at $30 \mathrm{~min}$ of exercise in the trial with the caffeinated bars. A recent study indicated that the presence of caffeine in a glucose beverage increased the rate of exogenous carbohydrate oxidation, most likely via a 
Caffeine and Cognitive Function during Exercise

stimulatory effect of caffeine on intestinal glucose absorption (31ㅂ) and a similar effect may account for our observation in the present study.

Paragraph 55 Other measures such as RPE, respiratory parameters and sweat loss were similar to those observed in other similar studies. For example, Kovacs et al. (17) reported no changes in sweat loss with caffeine compared with non caffeine trials during $1 \mathrm{~h}$ of cycling at $70 \% \dot{\vee} \mathrm{O}_{2}$ max. A review from Magkos and Kavouras (23) stated that studies have found no significant effects of caffeine on oxygen uptake during exercise at a given fixed work rate. Findings suggest that effects may have occurred through reduction of central fatigue mechanisms. This findings are similar to several earlier studies $(5,6)$.

Paragraph 56 Limitations of the present study are that blinding of the caffeine containing bar may have failed. However, discourse analyses revealed that the central alerting effects rather than physical characteristics of the treatment were conducive in this and were probably only recognized at the end -or during the later stages- of the trial.

Paragraph 57 In conclusion, caffeine in a performance bar can significantly improve physical and mental ability during and after endurance exercise. It is notable that the improvement in cognitive performance with caffeine was still present following the ride to exhaustion even though participants exercised for longer in the trial with the caffeinated bars. The results have important implications for all endurance sports performance in which concentration, complex information processing and vigilance play a major role.

\section{Acknowledgements}

We would like to acknowledge Chris Racey, Rebecca Prime, Will Eastwood and all the participating athletes for their invaluable help with data-collection. We are also grateful to $\mathrm{Dr}$ 
Caffeine and Cognitive Function during Exercise

Dominik Grathwohl, Dr Jacquez Decombaz and Dr Lionel Philippe from the Nestlé Research Center in Lausannne for their advice and input. This study was fully sponsored by Nestlé,

Switzerland. The results of the present study do not constitute endorsement by ACSM. 
Caffeine and Cognitive Function during Exercise

\section{REFERENCES}

1. Bandelow B, Hogervorst E. Diagnosing dementia using computerized test. JEAAM. 2006; March: 1-3.

2. Borg G. Borg's Perceived Exertion and Pain Scales. Champaign: Human Kinetics; 1998. (Figure 7.3, p. 49).

3. Bruce CR, Anderson ME, Fraser SF, Stepto NK, Klein R, Hopkins WG, Hawley JA. Enhancement of 2000-m rowing performance after caffeine ingestion. Med Sci Sports Exerc. 2000; 32:1958-63.

4. Crowe MJ, Leicht AS, Spinks WL. Physiological and cognitive responses to caffeine during repeated, high-intensity exercise. Int J Sport Nutr Exerc Metab. 12006; 6:52844.

5. Doherty M, Smith PM, Hughes MG, Davidson RCR. Caffeine lowers perceptual responses and increases power output during high-intensity cycling. J Sports Sci. $2004 ; 22: 637-43$

6. Graham TE. Caffeine and exercise: Metabolism, endurance and performance. Sports Med. 2001; 31:785-807.

7. Graham TE, Spriet LL. Metabolic, catecholamine, and exercise performance responses to various doses of caffeine. J Appl Physiol. 1995; 78:867-74 .

8. Greer F, Mclean C, Graham TE. Caffeine, performance, and metabolism during repeated Wingate exercise tests. J Appl Physiol. 1998; 85:1502-8.

9. Greer F, Morales J, Coles M. Wingate performance and surface EMG frequency variables are not affected by caffeine ingestion. Appl Physiol Nutr Metab. 2006; 31:597-603. 
Caffeine and Cognitive Function during Exercise

10. Grego F, Vallier JM, Collardeaus M, et al. Effects of long duration exercise on cognitive function, blood glucose, and counterregulatory hormones in male cyclists. Neurosci Lett. 2004; 364:76-80.

11. Herbert M, Foulds J, Fife-Schaw C. No effect of cigarette smoking on attention or mood in non-deprived smokers Addiction. 2001; 96:1349-56.

12. Hogervorst E, Riedel WJ, Jeukendrup AE, Jolles J. Cognitive performance after strenuous physical exercise. Percep Motor Skills. 1996; 83:479-88.

13. Hogervorst E, Riedel WJ, Kovacs E, Brouns F, Jolles J. Caffeine improves cognitive performance after strenuous physical exercise. Int J Sports Med. 1999; 20:354-61.

14. Jackman M, Wendling P, Friars D, Graham TE. Metabolic catecholamine, and endurance responses to caffeine during intense exercise. J Appl Physiol. 1996; 81:1658-63.

15. Kalmar JM, Cafarelli E. Caffeine: a valuable tool to study central fatigue in humans? Exerc Sport Sci Rev. 2001; 32:143-7.

16. Kennedy DO, Scholey AB. A glucose-caffeine 'energy drink' ameliorates subjective and performance deficits during prolonged cognitive demand Appetite. 2004; 42:3313.

17. Kovacs EM, Stegen JHCH, Brouns F. Effect of caffeinated drinks on substrate metabolism, caffeine excretion, and performance. J Appl Physiol. 1998; 85:709-15.

18. Kruk B, Chmura J, Krzeminski K, Ziemba AW, Nazar K, Pekkarinen H, KaciubaUscilko $\mathrm{H}$. Influence of caffeine, cold and exercise on multiple choice reaction time. Psychopharmacol (Berl). 2001; 157:197-201. 
Caffeine and Cognitive Function during Exercise

19. Lieberman HR, Wurtman RJ, Emde GG, Roberts C, Coviella ILG. The effects of low doses of caffeine on human performance and mood. Psychopharmacol. 1987; 92:30812.

20. Lorino AJ, Lloyd LK, Crixell SH, Walker JL. The effects of caffeine on athletic agility. J Strength Cond Res. 2006; 20:851-4.

21. Lorist MM, Snel J, Kok A. Influence of caffeine on information processing stages in well rested and fatigued subjects. Psychopharmacol. 1994; 113:411-21.

22. Lorist MM, Snel J, Kok A, Mulder G. Influence of caffeine on selective attention in well-rested and fatigued subjects. Psychophysiol. 1994; 31:525-34.

23. Magkos, F., And S. A. Kavouras. Caffeine use in sports, pharmacokinetics in man, and cellular mechanisms of action. Crit. Rev. Food Sci. Nutr. 45:535-562, 2005.

24. Pasman WJ, Van Baak MA, Jeukendrup AE, De Haan A. The effect of different dosages of caffeine on endurance performance time. Int J Sports Med. 1995; 16:22530.

25. Rogers NL, Dinges DF. Caffeine: implications for alertness in athletes. Clin J Sports Med. 2005; 24(2):e1-13, x-xi.

26. Schmitt JA, Hogervorst E, Vuurman EF, Jolles J, Riedel WJ. Memory functions and focussed attention in middle-aged and elderly subjects are unaffected by a low, acute dose of caffeine. J Nutr Health Aging. 2003; 7:301-3.

27. Stroop JR. The Stroop Test. J Exp Psychol. 1935; 18:643-62.

28. Tarnopolsky MA. Caffeine and endurance performance. Sports Med. 1994; 18:10925. 
Caffeine and Cognitive Function during Exercise

29. Welsh RS,. Davis MJ, Burke JR, Williams HG. Carbohydrates and physical/mental Formatted: Bullets and Numbering performance during intermittent exercise to fatigue. Med Sci Sports Exerc. 2002; Formatted: Font: Italic 34(4): 723-731.

Formatted: Font: Not Italic

29.30. Williams JH. Caffeine, neuromuscular function and high-intensity exercise Formatted: Bullets and Numbering performance J Sports Med Phys Fitness. 1991; 31:481-9.

30.31. Yeo SF, Jentjens RLPG, Wallis GA, Jeukendrup AE. Caffeine increases exogenous carbohydrate oxidation during exercise. J Appl Physiol. 2005; 99:844-50.

31.32. Yeomans MR, Ripley T, Davies LH, Rusted JM, Rogers P. Effects of caffeine on performance and mood depend on the level of caffeine abstinence. Psychopharmacol. 2002; 164:241-9. 
Caffeine and Cognitive Function during Exercise

\section{FIGURE LEGENDS}

Figure 1. Schematic representation of a test day, showing exercise protocol, key assessments and product administration. See text for full description.

Figure 2. Treatment and time effects on speed and accuracy on both Stroop test levels. The adjusted $\mathrm{P}$ values for the contrasts $\mathrm{CAF}-\mathrm{CHO}, \mathrm{BEV}-\mathrm{CAF}$ and $\mathrm{BEV}-\mathrm{CHO}$ are shown as CCO, CAF and CHO, respectively. Significance codes of the post-hoc comparisons (adjusted for 3 comparisons at each time point) are $*$ for $\mathrm{P}<0.05$, ** for $\mathrm{P}<0.01$ and $* * *$ for $\mathrm{P}<0.001$.

Figure 3. Treatment and time effects on speed, TP rate and miss rate on the RVIP test. The resulting adjusted $\mathrm{P}$ values for the contrasts $\mathrm{CAF}-\mathrm{CHO}, \mathrm{BEV}-\mathrm{CAF}$ and $\mathrm{BEV}-\mathrm{CHO}$ are shown as CCO, CAF and CHO, respectively. Significance codes of the post-hoc comparisons (adjusted for 3 comparisons at each time point) are * for $\mathrm{P}<0.05$, ** for $\mathrm{P}<0.01$ and $* * *$ for $\mathrm{P}<0.001$.

Figure 4. Treatment and time effects on speed and accuracy on the complex level of the Visual Search Test. The resulting adjusted $\mathrm{P}$ values for the contrasts CAF-CHO, BEV-CAF and BEV-CHO are shown as CCO, CAF and CHO, respectively. Significance codes of the post-hoc comparisons (adjusted for 3 comparisons at each time point) are * for $\mathrm{P}<0.05$, ** for $\mathrm{P}<0.01$ and $* * *$ for $\mathrm{P}<0.001$. 
Caffeine and Cognitive Function during Exercise

Figure 5. Time to exhaustion (sec) according to trials. * significantly different from BEV trial. \# significantly different from $\mathrm{CHO}$ trial $(\mathrm{P} \leq 0.05)$. 